\title{
Neutrophil extracellular traps are not produced in pediatric patients with one-lung ventilation: a prospective, single-center, observational study
}

\author{
Yingyi Xu ${ }^{1}$, Bingtai $\mathrm{Lu}^{2}$, Na Zhang ${ }^{1}$, Yufeng Liang ${ }^{3}$, Ying Gao ${ }^{1}$, Xiaoxin Ye ${ }^{4}$, Wei Liu ${ }^{5}$ \\ ${ }^{1}$ Department of Anesthesiology, Guangzhou Women and Children's Medical Center, Guangzhou Medical University, Guangzhou, China; ${ }^{2}$ Institute \\ of Pediatrics, Guangzhou Women and Children's Medical Center, Guangzhou Medical University, Guangzhou, China; ${ }^{3}$ Pediatric Intensive Care \\ Unit, Guangzhou Women and Children's Medical Center, Guangzhou Medical University, Guangzhou, China; ${ }^{4}$ School of Computer Science and \\ Engineering, The University of New South Wales, Sydney, Kensington, Australia; ${ }^{5}$ Department of Pediatric Surgery, Guangzhou Women and \\ Children's Medical Center, Guangzhou Medical University, Guangzhou, China \\ Contributions: (I) Conception and design: Y Xu, B Lu; (II) Administrative support: W Liu, Y Liang; (III) Provision of study materials or patients: Y \\ Xu, N Zhang, Y Liang, W Liu; (IV) Collection and assembly of data: Y Xu, N Zhang; (V) Data analysis and interpretation: B Lu, Y Gao, X Ye; (VI) \\ Manuscript writing: All authors; (VII) Final approval of manuscript: All authors. \\ Correspondence to: Wei Liu, M.M. Department of Pediatric Surgery, Women and Children's Medical Center, Guangzhou Medical University, Renmin \\ Middle Road 318, Yuexiu District, Guangzhou 510623, China. Email: liuwei19610624@126.com
}

Background: One-lung ventilation (OLV) may cause lung injury and induce pulmonary pro-inflammation; this ventilator-induced lung injury is associated with neutrophil infiltration. The infiltrated neutrophils release neutrophil extracellular traps (NETs), which are associated with tissue damage. It is not known whether NETs are involved in the pathogenesis of one-lung injury and if they could be a potential therapeutic target. In the present study, we quantified NETs in bronchoalveolar lavage fluid from pediatric patients who underwent OLV and assessed their relationship with prognosis.

Methods: Eighteen patients with congenital pulmonary cysts or pulmonary sequestration were enrolled in this prospective monocentric study. Myeloperoxidase (MPO) levels, NET markers [i.e., citrullinated histone-3 (CH-3) and free double-stranded DNA (dsDNA)], and inflammatory cytokine levels in bronchoalveolar lavage fluid were assessed. Continuous variables were compared using the paired t-test. The association of NET concentration in bronchoalveolar lavage fluid and clinical parameters was assessed using linear regression analyses.

Results: dsDNA concentration in bronchoalveolar lavage fluid was higher after OLV than before OLV in both the affected lung $(0.23 \pm 0.30$ vs. $0.97 \pm 1.05, \mathrm{P}<0.05)$ and the healthy lung $(0.28 \pm 0.19$ vs. $2.45 \pm 2.23$, $\mathrm{P}<0.05)$. However, there were no significant differences in concentrations of $\mathrm{MPO}, \mathrm{CH}-3$, and inflammatory cytokines before and after OLV. Serum interleukin (IL)-6 concentration was higher after OLV than before $(\mathrm{t}=-3.222, \mathrm{P}=0.007)$. Moreover, no associations between dsDNA concentration in bronchoalveolar lavage fluid and the duration of postoperative mechanical ventilation, postoperative hospital stay, and chest highresolution computed tomography score were observed. The durations of OLV, anesthesia, and operation, as well as the amount of blood loss, had no significant influence on postoperative dsDNA concentration in bronchoalveolar lavage fluid.

Conclusions: NETs in bronchoalveolar lavage fluid are not involved in patients who undergo OLV.

Keywords: Neutrophil extracellular trap (NET); bronchoalveolar lavage; lung injury; one-lung ventilation

Submitted Sep 14, 2020. Accepted for publication Oct 28, 2020.

doi: 10.21037/tp-20-337

View this article at: http://dx.doi.org/10.21037/tp-20-337 


\section{Introduction}

One-lung ventilation (OLV) improves the success rate of complex intrathoracic surgery using minimally invasive techniques. However, OLV could induce injuries to the lungs at both the ventilation and collapsed sides (1), and is associated with postoperative pulmonary complications in about $20 \%$ of patients who undergo lung resection (2). Lung collapse results in increased reactive oxygen species (ROS) and edema of the alveolar-capillary membrane (3). There were complications following OLV, including hypoxemia, carbon dioxide retention, recurrent pulmonary edema. atelectasis Lung re-expansion and re-ventilation after $1-3 \mathrm{~h}$ of OLV has been found to result in cytokine release, protein extravasation, and neutrophil recruitment into the alveolus. Neutrophil infiltration and alveolar structural damage increase with the duration of lung collapse (4). Although the mechanisms of lung injury remain poorly understood, neutrophil infiltration and a variety of inflammatory mediators have been reported to be associated with injury (5). In OLV-induced histomorphological lung injury, polymorphonuclear leucocytes are the most abundant effector cells. They are involved in lung injury and are involved in the dilation and hemorrhage of pulmonary vessels and inflammatory cell infiltration in the alveolar interstitium.

Neutrophil extracellular traps (NETs) are extracellular fibers composed of DNA, histones, and granule-derived proteins, such as elastase and myeloperoxidase (MPO), which are released by neutrophils during NET formation (NETosis) (6,7). NETs can trap and kill extracellular pathogens. However, experimental data have demonstrated that NETs may also cause tissue injury to the host by accumulated extracellular histones (the major component of NETs), which are highly toxic and may induce respiratory failure when infused intravenously (8). In addition, NETs have been detected in the lungs of mice subjected to endotoxemia or aggressive mechanical ventilation $(9,10)$. NETosis is a mode of cell death independent of apoptosis or necrosis. The mechanism of NETosis is not completely understood, but appears to include both ROS-dependent and -independent pathways, resulting in citrullination of histones (a key marker of NETosis) and enabling chromatin decondensation (11). Several known inducers of NETosis, including the cytokines interleukin (IL)- 8 and IL-1 $\beta$, have been shown to be increased in models of lung injury associated with mechanical ventilation (10). NETs were also generated in VILI and pathogenic in a mouse model of VILI, and their formation was partially dependent on TLR4 (12). The detrimental role of NETs has been reported in various clinical conditions, such as autoimmune disorders (13), thrombosis (14), and cystic fibrosis (15), but NET production in the lungs and blood in people who undergo OLV has not been comprehensively explored. It is not known whether NETs are involved in the pathogenesis of OLV-induced lung injury in humans and if they could be a potential therapeutic target. Like adults, children also need to undergo OLV during thoracic surgery. However, the physiological responses in pediatric patients are not well elucidated.

In the present study, we hypothesized that NETs are produced in the lungs of patients with OLV and are associated with regional neutrophil infiltration, and that NET concentration in bronchoalveolar lavage fluid is associated with clinical outcomes, based on the duration of postoperative mechanical ventilation, postoperative hospital stay, and chest high-resolution computed tomography (HRCT). We quantified NET production in bronchoalveolar lavage fluid in immunocompetent patients with OLV to test these hypotheses. We present the following article in accordance with the STROBE reporting checklist (available at http://dx.doi.org/10.21037/tp-20-337).

\section{Methods}

\section{Study design}

The present study was a prospective, single-center, observational study and was approved by the Ethics Committee of Guangzhou Women and Children's Medical Center (approval no. 2017070501, July 21, 2017). The study protocol was registered with Chinese Clinical Trials Registry (ChiCTR1900027099, supplementary registration) on October 31, 2019 by Yingyi Xu (principal investigator) prior to patient enrollment. Consecutive pediatric patients scheduled for selective resection of congenital pulmonary cysts or pulmonary sequestration between June 2016 and March 2017 were eligible for inclusion in the study. Written informed consent was obtained by the children's guardians. The present study was conducted in compliance with the Good Clinical Practice guidelines and the applicable Consolidated Standards of Reporting Trials guidelines. All procedures performed in this study involving human participants were in accordance with the Declaration of Helsinki (as revised in 2013).

No statistical power calculation was conducted prior to 
the study because of the lack of data regarding lung NET production in humans during OLV at the time the study was designed.

\section{Patient selection and data collection}

Patients who were classified as American Society of Anesthesiologists (ASA) physician status I or II, aged 1-6 years, and scheduled for selective resection of congenital pulmonary cysts or pulmonary sequestration were enrolled in the present study. Patients with heart diseases, immunosuppression, a history of inflammation, or coagulation dysfunction were excluded. All patients were monitored by electrocardiography, pulse oximetry, invasive blood pressure recording, and bispectral index values. For fluid supplementation, $3-5 \mathrm{~mL} / \mathrm{kg} / \mathrm{h}$ crystalloid was administered; $2 \mathrm{mg} / \mathrm{kg}$ propofol, $0.3 \mathrm{mcg} / \mathrm{kg}$ sufentanil, and $0.2 \mathrm{mg} / \mathrm{kg}$ cisatracurium were intravenously administered for anesthetic induction. A bronchial occluder was successfully inserted after tracheal intubation, and the location was confirmed with a fiber bronchoscope. Volume-controlled mechanical ventilation was performed after tracheal intubation with a tidal volume of $8 \mathrm{~mL} / \mathrm{kg}$ predicted body weight (PBW) during 2-lung ventilation (TLV) and $6 \mathrm{~mL} / \mathrm{kg}$ PBW during OLV. The inspired oxygen fraction was set to 0.5 during TLV and 1.0 during OLV. Respiratory rate was set to $20-25$ breaths/min and adjusted to maintain partial pressure of carbon dioxide in end-expiratory gas between 35 and $45 \mathrm{mmHg}$. Positive endexpiratory pressure was maintained at $5 \mathrm{cmH}_{2} \mathrm{O}$. Anesthesia was maintained with $2-3 \%$ sevoflurane and cisatracurium. The depth of anesthesia was maintained with a bispectral index between 40 and 60 (Covidien, St. Louis, MO, USA) by changing the infusion speed of propofol and sevoflurane. Sedation and mechanical ventilation weaning followed standardized protocols. While the patient was mechanically ventilated in the intensive care unit, primary sedative agents fentanyl $(2 \mathrm{mcg} / \mathrm{kg} / \mathrm{h})$ and midazolam $(0.1 \mathrm{mg} / \mathrm{kg} / \mathrm{h})$ were used, and Ramsay Sedation Scale score was maintained at 3-4. Midazolam and fentanyl infusions were discontinued at least $3 \mathrm{~h}$ prior to planned extubation. Demographic, clinical, and laboratory variables were recorded during the whole procedure, including OLV, intrathoracic surgery, anesthesia, and estimated blood loss. Other recorded variables included postoperative complications, chest HRCT score (16), and the duration of mechanical ventilation and postoperative hospital stay.

\section{NET and cytokine concentration measurements in bronchoalveolar lavage fluid and blood}

Bronchoalveolar lavage fluid was collected from all patients after tracheal intubation and following surgery. A blood sample for the measurement of cytokine concentration was obtained at the same time. NETs were quantified by measuring double-stranded DNA (dsDNA) and citrullinated histone-3 (CH-3) in bronchoalveolar lavage fluid samples. dsDNA in the bronchoalveolar lavage fluid supernatant was quantified using Quant-iT Picogreen (ThermoFisher Waltham, MA, USA), following the manufacturer's protocol. CH-3 was quantified using a previously described capture enzyme-linked immunosorbent assay (16). Optical density was measured at $405 \mathrm{~nm}$. Data obtained from a strongly positive serum were used to draft the standard curve. Samples were interpolated from the standard curve using the sigmoidal dose-response equation, and results were expressed in arbitrary units (detection range, 4-1,000 arbitrary units). Optical density values outside the detection range of arbitrary units (i.e., values $<4$ and $>1,000$ arbitrary units) were replaced by the threshold value. Serum and bronchoalveolar lavage fluid concentrations of the inflammatory markers involved in neutrophil chemotaxis and activation [IL-6, IL- $1 \beta$, tumor necrosis factor- $\alpha$ $(\mathrm{TNF}-\alpha)$ ] were measured using the Human Magnetic Luminex Assay (R\&D Systems, Minneapolis, MN, USA). In addition, MPO concentration in serum and bronchoalveolar lavage fluid were also measured by Western blotting.

\section{Statistical analysis}

Continuous variables were reported as medians (1st-3rd quartiles) or means \pm standard deviations, as appropriate, and compared using the paired $t$-test. Normality of the data was tested using the Shapiro-Wilk normality test. Categorical variables were reported as numbers and percentages (or $95 \%$ confidence intervals), and compared using the $\chi^{2}$-test or Fisher's exact test, as appropriate. Missing data were not replaced. No outlier value was excluded from the current dataset. The relationship between NET concentration in bronchoalveolar lavage fluid and clinical characteristics was further assessed using linear regression analyses. All statistical analyses were 2 tailed, and $\mathrm{P}<0.05$ was considered statistically significant. Analyses were conducted using SPSS version 15.0 (SPSS, NCSS, Utah, USA). 
Table 1 Demographic characteristics of the 18 patients who underwent OLV for thoracic surgery

\begin{tabular}{ll}
\hline Characteristics & Data \\
\hline Sex [cases (\%)] & $8(44.4)$ \\
Male & $10(55.6)$ \\
Female & $21[10-36]$ \\
Age at surgery [months; median (1st-3rd quartiles)] & $10.8(8.5-12)$ \\
Weight at surgery [kg; median (1st-3rd quartiles)] & 82 [78-92] \\
Height at surgery [cm; median (1st-3rd quartiles)] & $15.57(14.71-17.23)$ \\
Body mass index at surgery [kg/cm²; median (1st-3rd quartiles)] & $18[100]$ \\
ASA I/II [cases (\%)] & 70 [60-120] \\
OLV duration [min; median (1st-3rd quartiles)] & 105 [90-120] \\
Surgery duration [min; median (1st-3rd quartiles)] & $180[150-210]$ \\
Anesthesia duration [min; median (1st-3rd quartiles)] & 75 (27.5-137.5) \\
Estimated blood loss [mL; median (1st-3rd quartiles)] & $7.5(5.0-10.5)$ \\
Mechanical ventilation duration [h; median (1st-3rd quartiles)]
\end{tabular}

OLV, one-lung ventilation; ASA, American Standards Association.

Table 2 Differences in CH-3, IL-6, IL-1 $\beta$, TNF- $\alpha$, MPO, and dsDNA between affected and healthy lungs before and after surgery

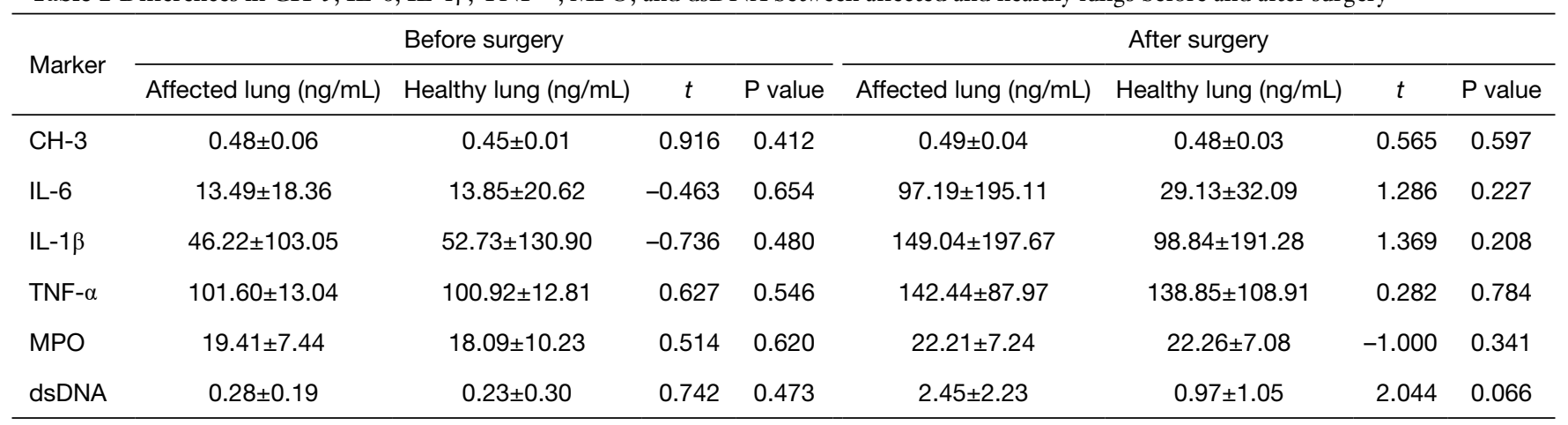

$\mathrm{CH}-3$, citrullinated histone-3; dsDNA, double-stranded DNA; IL, interleukin; MPO, myeloperoxidase; TNF- $\alpha$, tumor necrosis factor- $\alpha$.

\section{Results}

\section{Patients' demographic and surgical characteristics}

A total of 20 patients were enrolled in the present study; 2 were excluded because of a lack of bronchoalveolar lavage fluid sample. Patients' demographic characteristics are summarized in Table 1.

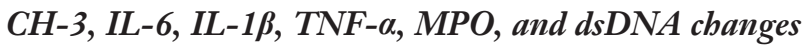 in bronchoalveolar lavage fluid from both lungs before and after surgery}

As shown in Table 2, no significant differences in CH-3,
IL-6, IL- $1 \beta$, TNF- $\alpha$, MPO, and dsDNA between the affected and healthy lungs were observed before and after surgery (all $\mathrm{P}>0.05$ ). As shown in Table 3, the dsDNA level after surgery was higher than that before surgery in both the affected lung $(\mathrm{P}=0.006)$ and the healthy lung $(\mathrm{P}=0.015)$.

\section{$C H-3, I L-6, I L-1 \beta, T N F-\alpha$, and MPO changes in serum before and after surgery}

As shown in Table 4, the IL-6 serum concentration after surgery was significantly higher than that before surgery $(\mathrm{t}=-3.222, \mathrm{P}=0.007)$. No other significant changes were observed. 
Table 3 CH-3, IL-6, IL-1 $\beta$, TNF- $\alpha$, MPO, and dsDNA changes before and after surgery in both affected and healthy lungs

\begin{tabular}{|c|c|c|c|c|c|c|c|c|}
\hline Marker & \multicolumn{4}{|c|}{ Affected lung } & \multicolumn{4}{|c|}{ Healthy lung } \\
\hline $\mathrm{CH}-3$ & $0.46 \pm 0.04$ & $0.49 \pm 0.04$ & -1.553 & 0.181 & $0.45 \pm 0.01$ & $0.48 \pm 0.04$ & -1.321 & 0.278 \\
\hline IL-6 & $12.94 \pm 17.51$ & $97.19 \pm 195.11$ & -1.410 & 0.189 & $13.85 \pm 20.62$ & $31.07 \pm 33.14$ & -1.419 & 0.190 \\
\hline IL-1 $\beta$ & $45.10 \pm 103.41$ & $151.86 \pm 186.57$ & -1.510 & 0.165 & $57.36 \pm 137.97$ & $98.62 \pm 191.39$ & -0.549 & 0.598 \\
\hline MPO & $18.66 \pm 7.48$ & $22.21 \pm 7.24$ & -2.120 & 0.060 & $18.09 \pm 10.23$ & $19.96 \pm 9.37$ & -0.520 & 0.615 \\
\hline dsDNA & $0.28 \pm 0.19$ & $2.45 \pm 2.23$ & -3.387 & $0.006^{*}$ & $0.23 \pm 0.30$ & $0.97 \pm 1.05$ & -2.870 & $0.015^{*}$ \\
\hline
\end{tabular}

*, statistically significant at $\mathrm{P}<0.05 . \mathrm{CH}-3$, citrullinated histone-3; dsDNA, double-stranded DNA; IL, interleukin; MPO, myeloperoxidase; TNF- $\alpha$, tumor necrosis factor- $\alpha$.

Table 4 CH-3, IL-6, IL-1 $\beta$, TNF- $\alpha$, and MPO changes in serum before and after surgery

\begin{tabular}{lcccc}
\hline Marker & Before surgery $(\mathrm{ng} / \mathrm{mL})$ & After surgery $(\mathrm{ng} / \mathrm{mL})$ & $t$ & \multicolumn{1}{c}{ P value } \\
\hline CH-3 & $2.66 \pm 0.94$ & $2.22 \pm 0.72$ & 1.620 & 0.149 \\
IL-6 & $9.49 \pm 1.81$ & $29.73 \pm 23.47$ & -3.222 & $0.007^{\star}$ \\
IL-1 $\beta$ & $5.93 \pm 1.04$ & $5.68 \pm 0.96$ & 0.783 & 0.447 \\
TNF- $\alpha$ & $132.74 \pm 8.31$ & $130.66 \pm 8.62$ & 0.893 & 0.388 \\
MPO & $5.83 \pm 4.22$ & $6.59 \pm 3.56$ & -0.496 & 0.628
\end{tabular}

*, statistically significant at $\mathrm{P}<0.05$. $\mathrm{CH}-3$, citrullinated histone-3; IL, interleukin; MPO, myeloperoxidase; TNF- $\alpha$, tumor necrosis factor- $\alpha$.

Table 5 Neutrophil extracellular trap and cytokine production in lungs before and after OLV

\begin{tabular}{|c|c|c|c|c|c|c|c|c|}
\hline Factor & \multicolumn{4}{|c|}{ Affected lung } & \multicolumn{4}{|c|}{ Healthy lung } \\
\hline $\mathrm{CH}-3$ & $0.46 \pm 0.04$ & $0.49 \pm 0.04$ & -1.553 & 0.181 & $0.45 \pm 0.01$ & $0.48 \pm 0.04$ & -1.321 & 0.278 \\
\hline IL-6 & $12.94 \pm 17.51$ & $97.19 \pm 195.11$ & -1.410 & 0.189 & $13.85 \pm 20.62$ & $31.07 \pm 33.14$ & -1.419 & 0.190 \\
\hline IL-1 $\beta$ & $45.10 \pm 103.41$ & $151.86 \pm 186.57$ & -1.510 & 0.165 & $57.36 \pm 137.97$ & $98.62 \pm 191.39$ & -0.549 & 0.598 \\
\hline MPO & $18.66 \pm 7.48$ & $22.21 \pm 7.24$ & -2.120 & 0.060 & $18.09 \pm 10.23$ & $19.96 \pm 9.37$ & -0.520 & 0.615 \\
\hline dsDNA & $0.28 \pm 0.19$ & $2.45 \pm 2.23$ & -3.387 & 0.006 & $0.23 \pm 0.30$ & $0.97 \pm 1.05$ & -2.870 & 0.015 \\
\hline
\end{tabular}

OLV, 1-lung ventilation; $\mathrm{CH}-3$, citrullinated histone-3; dsDNA, double-stranded DNA; IL, interleukin; MPO, myeloperoxidase; TNF- $\alpha$, tumor necrosis factor- $\alpha$.

\section{NET and cytokine production in the lungs before and after $O L V$}

The dsDNA concentration in bronchoalveolar lavage fluid after OLV was higher than that before OLV in both the affected lung $(\mathrm{P}=0.006)$ and the healthy lung $(\mathrm{P}=0.015)$ (Table 5). No significant changes in $\mathrm{CH}-3$ and cytokine production before and after OLV were observed in both lungs.

\section{$C H-3, I L-6, I L-1 \beta, T N F-\alpha$, and MPO production in serum before and after $O L V$}

The serum concentration of IL-6 after OLV was 
Table 6 Cytokine concentration in serum before and after OLV

\begin{tabular}{lcccc}
\hline Factor & Before OLV $(\mathrm{ng} / \mathrm{mL})$ & After OLV $(\mathrm{ng} / \mathrm{mL})$ & $t$ & P value \\
\hline CH-3 & $2.66 \pm 0.94$ & $2.22 \pm 0.72$ & 1.620 & 0.149 \\
IL-6 & $9.49 \pm 1.81$ & $29.73 \pm 23.47$ & -3.222 & 0.007 \\
IL-1 $\beta$ & $5.93 \pm 1.04$ & $5.68 \pm 0.96$ & 0.783 & 0.447 \\
TNF- $\alpha$ & $132.74 \pm 8.31$ & $130.66 \pm 8.62$ & 0.893 & 0.388 \\
MPO & $5.83 \pm 4.22$ & $6.59 \pm 3.56$ & -0.496 & 0.628 \\
\hline
\end{tabular}

OLV, one-lung ventilation; $\mathrm{CH}-3$, citrullinated histone-3; IL, interleukin; MPO, myeloperoxidase; TNF- $\alpha$, tumor necrosis factor- $\alpha$.
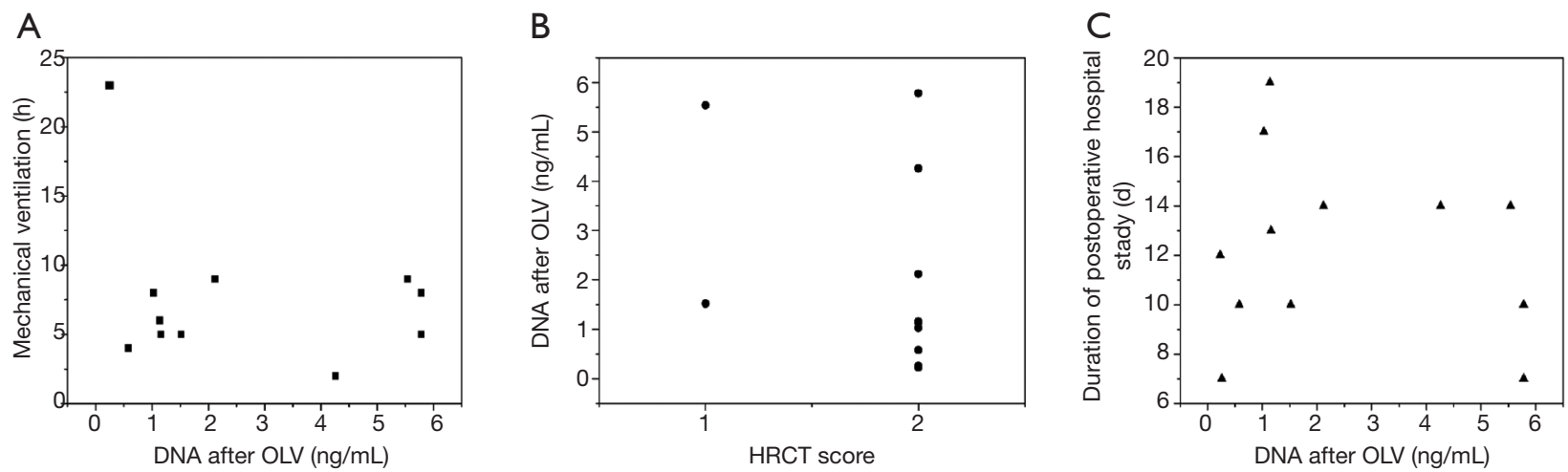

Figure 1 Relationship between double-stranded DNA (dsDNA) concentration in bronchoalveolar lavage fluid and postoperative outcomes of patients. (A) Relationship between dsDNA concentration and the duration of postoperative mechanical ventilation. (B) Relationship between dsDNA concentration and chest high-resolution computed tomography (HRCT) score. (C) Relationship between dsDNA concentration and the duration of postoperative hospital stay. $\mathbf{m}$, scatter plot of dsDNA concentration and the duration of postoperative mechanical ventilation; •, scatter plot of dsDNA concentration and chest high-resolution computed tomography (HRCT) score; $\boldsymbol{\Delta}$, scatter plot of dsDNA concentration and the duration of postoperative hospital stay. OLV, one-lung ventilation.

significantly higher than that before OLV ( $\mathrm{t}=-3.222$, $\mathrm{P}=0.007)$. No significant changes in other cytokines before and after OLV were observed (Table 6).

\section{Postoperative outcomes of patients with different dsDNA concentrations in bronchoalveolar lavage fluid}

The postoperative outcomes of patients with different dsDNA concentrations in bronchoalveolar lavage fluid were compared using linear regression or Spearman's analysis. No significant relationship between dsDNA concentration and the duration of postoperative mechanical ventilation, chest HRCT score, or duration of postoperative hospital stay was observed (Figure 1, Table 7).

\section{Discussion}

OLV is commonly used in thoracic surgery. It can isolate the lungs to enable ventilation by the healthy lung, while preventing blood effusion and secretion from the affected lung into the healthy bronchus. OLV enables access during surgery. However, OLV can also negatively impact respiratory physiology, hemodynamics, and respiratory mechanics of the lungs and is a risk factor for acute lung injury, which may progress to acute respiratory distress syndrome (ARDS) (17). The occurrence rate of acute lung injury and ARDS after pulmonectomy has been reported to be $2 \%$, and that of acute lung injury is $4-15 \%$ (18). Acute lung injury and ARDS are the leading causes of death after thoracic surgery, significantly reducing the 1 -year survival rate from $92 \%$ to $56 \%(19)$. Therefore, elucidating the mechanism of lung injury caused by OLV is essential for reducing complications after thoracotomy and improving patient safety.

At present, none of the tested pharmacological interventions for lung injury, including, but not limited 
Table 7 Influence of dsDNA after OLV on clinical outcome

\begin{tabular}{|c|c|c|c|}
\hline \multicolumn{4}{|c|}{ dsDNA in contralateral lung } \\
\hline$P$ value & 0.364 & 0.906 & 0.650 \\
\hline \multicolumn{4}{|c|}{ dsDNA in ipsilateral lung } \\
\hline $\mathrm{P}$ value & 0.187 & 0.873 & 0.319 \\
\hline
\end{tabular}

dsDNA, double-stranded DNA; HRCT, high-resolution computed tomography; OLV, one-lung ventilation.

to, steroids (20), $\beta 2$-agonists (21), and or statins (22), has demonstrated significant survival benefit. The identification of disease severity biomarkers that would be potential therapeutic targets is therefore important for developing novel treatment for ARDS.

The major findings of the present study on pediatric patients who underwent OLV for thoracic surgery were as follows: (I) dsDNA concentration in bronchoalveolar lavage fluid increased after OLV in both lungs, but MPO and $\mathrm{CH}-3$ concentrations did not change, suggesting that NETs are not produced during OLV; and (II) the dsDNA concentration in bronchoalveolar lavage fluid was not significantly associated with postoperative mechanical ventilation duration, chest HRCT score, and duration of postoperative hospital stay.

Previous studies have demonstrated that mechanical ventilation may induce NET production and lung injury (12), and that neutrophil infiltration is involved in this process (15). As a type of mechanical ventilation, OLV can also cause neutrophil infiltration and ventilatorrelated lung injury. We speculated that NETs may also be produced during OLV. In the present study, we measured NET levels in bronchoalveolar lavage fluid samples from a relatively homogeneous cohort of 18 immunocompetent patients who underwent OLV. The dsDNA concentration in these samples was significantly elevated after OLV. This may be explained by the detection of dsDNA from necrotic leucocytes, which are not involved in the production of NETs. Other NET markers (MPO and $\mathrm{CH}-3$ ) were not elevated, suggesting the absence of NET production in the lungs and blood during OLV, which is inconsistent with our hypothesis. These findings may be due to several reasons. First, the production of NETs is caused by traumatic ventilation. In a previous mouse model of ventilationinduced lung injury (12), 1 group of mice underwent 1-h traumatic mechanical ventilation with a maximum inspiratory pressure of $45 \mathrm{cmH}_{2} \mathrm{O}$, and the other group of mice underwent 2 -h ventilation with a mild terminal inspiratory pressure of $15-20 \mathrm{cmH}_{2} \mathrm{O}$. NETs could only be detected in the lungs of mice with traumatic mechanical. In the present study, we adopted the protective ventilation strategy, which may not be sufficient to stimulate the production of NETs. Second, the duration of surgery and OLV was limited. The relationship between NET production and ventilation duration is unclear. Rossaint et al. detected NETs in the short-term ventilator-induced lung injury (VILI) model after traumatic ventilation, suggesting that long-term OLV may not induce NET production (23). The most important factor of NET production should be the degree of traumatic ventilation. We found that the dsDNA concentration was not related to the duration of ventilation, surgery, and anesthesia. In surgical patients with OLV, protective ventilation strategies are necessary. The impact of long-term OLV on NET production needs to be further investigated.

Recent studies have shown contradictory results of NETs in the pathogenesis of mechanical ventilation-related lung injury in mice. Brinkmann et al. demonstrated that NETs directly affect the severity of VILI and contribute to disease progression (7). In contrast, Yildiz et al. showed that NETs formed in the injured lung may have a limited role in the pathogenesis of VILI, indicating that reducing neutrophil dsDNA production could not reduce the degree of lung injury (11). In addition, some previously published studies found that that lung injury was not induced by NETs, and did not support the hypothesis that neutrophils are harmful in ARDS (24). NETs have been shown to play a protective role in early-stage ARDS accompanied with pneumonia, which is consistent with the antibacterial function of neutrophils through NETs. NETs can also 
capture and kill a variety of pathogens, such as Salmonella, Typhoid, Staphylococcus aureus, and Candida albicans (25). The mechanisms of lung injury by OLV include four hands: barotrauma, volutrauma, atelectrauma and hypoxia injury associated with reperfusion. These factors result in biotrauma which induced by cytokines and inflammatory mediators.

The present study has some limitations. First, the present study was a monocentric study and included a homogeneous cohort of patients with OLV, therefore limiting its external validity. Second, the number of patients in the cohort was relatively low, and the study was not designed based on power calculations to observe differences between patients with high and low NET production in bronchoalveolar lavage fluid, which limited outcome observations. Third, 2 patients with MPO concentrations exceeding the detection range were excluded from the statistical analyses. If the threshold value was used, the accuracy of statistical analyses might be improved. Fourth, the patients involved in the present study were pediatric patients with mediastinal cysts or diaphragmatic hernias; the patients had excellent lung function and no lung disease prior to surgery. However, the effect of OLV on NET production in patients with preoperative lung infection prior to surgery or tumors that require lung surgery is unclear. Therefore, further studies on a more diverse population are warranted.

\section{Acknowledgments}

Funding: None.

\section{Footnote}

Reporting Checklist: The authors have completed the STROBE reporting checklist. Available at http://dx.doi. org/10.21037/tp-20-337

Data Sharing Statement: Available at http://dx.doi. org/10.21037/tp-20-337

Conflicts of Interest: All authors have completed the ICMJE uniform disclosure form (available at http://dx.doi. org/10.21037/tp-20-337). The authors have no conflicts of interest to declare.

Ethical Statement: The authors are accountable for all aspects of the work in ensuring that questions related to the accuracy or integrity of any part of the work are appropriately investigated and resolved. The present study was approved by the Ethics Committee of Guangzhou Women and Children's Medical Center (approval no. 2017070501, July 21, 2017). All procedures performed in this study involving human participants were in accordance with the Declaration of Helsinki (as revised in 2013). Written informed consent was obtained by the children's guardians.

Open Access Statement: This is an Open Access article distributed in accordance with the Creative Commons Attribution-NonCommercial-NoDerivs 4.0 International License (CC BY-NC-ND 4.0), which permits the noncommercial replication and distribution of the article with the strict proviso that no changes or edits are made and the original work is properly cited (including links to both the formal publication through the relevant DOI and the license). See: https://creativecommons.org/licenses/by-nc-nd/4.0/.

\section{References}

1. Lohser J, Slinger P. Lung injury after one-lung ventilation: a review of the pathophysiologic mechanisms affecting the ventilated and the collapsed lung. Anesth Analg 2015;121:302-18.

2. Grande B, Ganter MT. What is the best strategy for onelung ventilation during thoracic surgery? J Thorac Dis 2018;10:6404-6.

3. Sivrikoz MC, Tuncozgur B, Cekmen M, et al. The role of tissue reperfusion in the re-expansion injury of the lungs. Eur J Cardiothorac Surg 2002;22:721-7.

4. Tekinbas C, Ulusoy H, Yulug E, et al. One-lung ventilation: for how long? J Thorac Cardiovasc Surg 2007;134:405-10.

5. Sakuma M, Khan MAS, Yasuhara S, et al. Mechanism of pulmonary immunosuppression: extrapulmonary burn injury suppresses bacterial endotoxin-induced pulmonary neutrophil recruitment and neutrophil extracellular trap (NET) formation. FASEB J 2019;33:13602-16.

6. Perkins GD, McAuley DF, Thickett DR, et al. The betaagonist lung injury trial (BALTI): A randomized placebocontrolled clinical trial. Am J Respir Crit Care Med 2006;173:281-7.

7. Brinkmann V, Reichard U, Goosmann C, et al. Neutrophil extracellular traps kill bacteria. Science 2004;303:1532-5.

8. Papayannopoulos V. Neutrophil extracellular traps in immunity and disease. Nat Rev Immunol 2018;18:134-47.

9. Xu J, Zhang X, Pelayo R, et al. Extracellular histones 
are major mediators of death in sepsis. Nat Med 2009;15:1318-21.

10. Saffarzadeh M, Juenemann C, Queisser MA, et al. Neutrophil extracellular traps directly induce epithelial and endothelial cell death: A predominant role of histones. PLoS One 2012;7:e32366.

11. Yildiz C, Palaniyar N, Otulakowski G, et al. Mechanical ventilation induces neutrophil extracellular trap formation. Anesthesiology 2015;122:864-75.

12. Li H,Pan P, Su X, et al. Neutrophil Extracellular Traps Are Pathogenic in Ventilator-Induced Lung Injury and Partially Dependent on TLR4. Biomed Res Int 2017;2017:8272504.

13. Corsiero E, Pratesi F, Prediletto E, et al. NETosis as Source of Autoantigens in Rheumatoid Arthritis. Front Immunol 2016;7:485.

14. Podolska MJ, Mahajan A, Hahn J, et al. Treatment with DNases rescues hidden neutrophil elastase from aggregated NETs. J Leukoc Biol 2019;106:1359-66.

15. Thålin C, Hisada Y, Lundström S, et al. Neutrophil Extracellular Traps: Villains and Targets in Arterial, Venous, and Cancer-Associated Thrombosis. Arterioscler Thromb Vasc Biol 2019;39:1724-38.

16. Khan MA, Ali ZS, Sweezey N, et al. Progression of Cystic Fibrosis Lung Disease from Childhood to Adulthood: Neutrophils, Neutrophil Extracellular Trap (NET) Formation, and NET Degradation. Genes (Basel) 2019;10:183.

17. Licker MJ, Widikker I, Robert J, et al. Operative mortality and respiratory complications after lung resection for cancer: impact of chronic obstructive pulmonary disease

Cite this article as: $\mathrm{Xu} \mathrm{Y,} \mathrm{Lu} \mathrm{B,} \mathrm{Zhang} \mathrm{N,} \mathrm{Liang} \mathrm{Y,} \mathrm{Gao} \mathrm{Y,}$ Ye X, Liu W. Neutrophil extracellular traps are not produced in pediatric patients with one-lung ventilation: a prospective, single-center, observational study. Transl Pediatr 2020;9(6):775783. doi: $10.21037 /$ tp-20-337 and time trends. Ann Thorac Surg 2006;81:1830-7.

18. Lohser J. Evidence-based management of one-lung ventilation. Anesthesiol Clin 2008;26:241-72.

19. Fernández-Pérez ER, Sprung J, Afessa B, et al. Intraoperative ventilator settings and acute lung injury after elective surgery: a nested case control study. Thorax 2009;64:121-7.

20. Steinberg KP, Hudson LD, Goodman RB, et al. Efficacy and safety of corticosteroids for persistent acute respiratory distress syndrome. N Engl J Med 2006;354:1671-84.

21. Perkins GD, McAuley DF, Thickett DR, et al. The betaagonist lung injury trial (BALTI): A randomized placebocontrolled clinical trial. Am J Respir Crit Care Med 2006;173:281-7.

22. McAuley DF, Laffey JG, O'Kane CM, et al. HARP-2 Investigators; Irish Critical Care Trials Group: Simvastatin in the acute respiratory distress syndrome. N Engl J Med 2014;371:1695-703.

23. Rossaint J, Herter JM, Van Aken H, et al. Synchronized integrin engagement and chemokine activation is crucial in neutrophil extracellular trap-mediated sterile inflammation. Blood 2014;123:2573-84.

24. Bendib I, de Chaisemartin L, Granger V, et al. Neutrophil Extracellular Traps Are Elevated in Patients with Pneumonia-related Acute Respiratory Distress Syndrome. Anesthesiology 2019;130:581-91.

25. Lögters T, Paunel-Görgülü A, Zilkens C, et al. Diagnostic accuracy of neutrophil-derived circulating free DNA (cf-DNA/NETs) for septic arthritis. J Orthop Res 2009;27:1401-7. 